\title{
The relevance of structural biology in studying molecules involved in parasite- host interactions - potential for designing new interventions
}

\author{
Lyndel Mason ${ }^{1}$, Parisa Amani ${ }^{2}$, Megan Cross ${ }^{1}$, Joshua Baker ${ }^{1}$, Ulla-Maja Bailey ${ }^{1}$, Malcolm K
} Jones $^{3,4}$, Robin B Gasser ${ }^{5}$ \& Andreas Hofmann ${ }^{1,5,6}$

\begin{abstract}
${ }^{1}$ Eskitis Institute, Griffith University, Nathan, Queensland, Australia
${ }^{2}$ Department of Chemistry, K. N. Toosi University of Technology, Tehran, Iran

${ }^{3}$ School of Veterinary Science, The University of Queensland, Gatton, Queensland, Australia ${ }^{4}$ QIMR Berghofer Medical Research Institute, Herston, Queensland, Australia

${ }^{5}$ Faculty of Veterinary Science, The University of Melbourne, Parkville, Victoria, Australia

${ }^{6}$ Queensland Tropical Health Alliance
\end{abstract}

This update: $06 / 06 / 14$

Word count: 7237 (47426)

Format: $\quad$ Aust. J. Chem.

Keywords: Activation-associated secreted proteins, Helminths, Saposin-like proteins, SCP/TAPS proteins 
New interventions against infectious diseases require a detailed knowledge and understanding of pathogen-host interactions and pathogeneses at the molecular level. The combination of the considerable advances in systems biology research with methods to explore the structural biology of molecules is poised to provide new insights into these areas. Importantly, exploring threedimensional structures of proteins is central to understanding disease processes, and establishing structure-function relationships assists in identification and assessment of new drug and vaccine targets.

Frequently, the molecular arsenal deployed by invading pathogens, and in particular parasites, reveals a common theme whereby families of proteins with conserved three-dimensional folds play crucial roles in infectious processes, but individual members of such families show high levels of specialisation which is often achieved through grafting particular structural features onto the shared overall fold. Accordingly, the applicability of predictive methodologies based on the primary structure of proteins or genome annotations is limited, particularly when thorough knowledge of molecular level mechanisms is required. Such instances exemplify the need for experimental threedimensional structures provided by protein crystallography, which remain an essential component of this area of research.

In the present article, we review two examples of key protein families recently investigated in our laboratories, which could represent intervention targets in the metabolome or secretome of parasites.

\section{Introduction}

Infectious diseases caused by eukaryotic pathogens represent a major disease burden to humans world-wide. In the absence of effective preventative approaches and new intervention strategies, this burden is likely to increase further, ${ }^{[1,2]}$ compounded by food and water shortages, economic crises, wars and climate change, particularly in disadvantaged countries. In addition, there is evidence of increasing problems with resistance in pathogens against a broad range of chemotherapeutic agents, compromising the treatment of infectious diseases. ${ }^{[3]}$ Moreover, in spite of major efforts, there are very few examples of success in developing new drugs and vaccines against eukaryotic pathogens, ${ }^{[4,5]}$ indicating that alternative methods are needed at a time of major advances in molecular and computer technologies. In our opinion, the development of new treatments requires a detailed understanding of pathogens, host interactions and the molecular processes of disease. For instance, knowledge of the three-dimensional structures of proteins with pivotal roles in disease, and the probing of their underlying biology are crucial for understanding the pathogenesis. Through establishing the relevant structure-function relationships, one can elucidate how individual proteins function, so that new ways of disrupting relevant pathways can be found, in order to facilitate the identification of new drug and vaccine targets.

This review covers recent investigations of two groups of socio-economically important pathogens of humans or other animals, the blood fluke (schistosome) Schistosoma mansoni, as well as hookworms (Necator americanus and Ancylostoma species). We provide an account of some key parasite proteins studied in our laboratories and their proposed roles in biological pathways, with a view towards assessing their suitability as intervention targets. Proteins providing structural integrity are another promising interference target, and have been investigated in schistosomes, ${ }^{[6]}$ tape worms ${ }^{[7]}$ as well as the protistan Giardia intestinalis (Metamonada), ${ }^{[8]}$ which is the cause of chronic diarrhoea in animals. Parasite target proteins involved in structural integrity have been reviewed in detail elsewhere. ${ }^{[9,10]}$

The technique of protein crystallography is poised to deliver much needed insights into the structure-function relationships of key pathogen molecules and their interactions with host proteins. 
Combined with cell and immuno-biological investigation, ${ }^{[11,12]}$ next-generation sequencing and transcriptomics methodologies, ${ }^{[13]}$ advanced modelling approaches ${ }^{[14]}$ and the probing of key proteins with small molecules, ${ }^{[15]}$ crystallography continues to provide the crucial information for rationale and target discovery.

Members of the genus Schistosoma pathogenic flatworms are responsible for chronic infections throughout Africa and many parts of the world. Schistosoma mansoni causes hepato-intestinal schistosomiasis due to the predilection of adult parasites to life in the mesenteric veins and its branches. Transition to parasitism in schistosomes involves major structural alterations of the apical membranes, and effective disruption of this layer might lead to death of these parasites within the host.

Hookworms are avid blood feeders. Adult hookworms inhabit the intestine of their hosts, where, by virtue of anatomic adaptations of their mouth-parts and strong secretory activity, they are able to imbibe quantities of their host blood. Prolonged infection with hookworms can give rise to severe sequelae associated with anaemia and can lead to developmental and cognitive impairment, particularly in children.

\section{Proteins in metabolic pathways: saposin-like proteins}

Saposin-like proteins (SAPLIPs) are a large and diverse family of proteins that interact with lipids. The proteins share little sequence homology amongst one another, but all have a conserved tertiary structure which defines the family. A subset of SAPLIPs, the mammalian saposins are activator proteins that facilitate degradation of glycosphingolipids and promote their hydrolytic processing by exohydrolases. ${ }^{[16]}$ Despite their similar structure, each saposin targets a distinct sphingolipid and enables its degradation by a partially overlapping set of enzymes. ${ }^{[17]}$ Saposins are further linked to the human immune response by loading hydrophobic lipid antigens from lysosomal membranes onto human CD1 molecules where they can be processed further by the immune system. ${ }^{[18]}$ In accord with these activities, saposins possess membrane binding and lipid transport properties, and they destabilise phospholipid vesicles and modulate the membrane structure in a detergent-like manner. ${ }^{[19]}$

SAPLIPs of blood flukes (or schistosomes) have attracted considerable interest, since they possess immunogenic properties; ${ }^{[20]}$ transcriptomic analyses have revealed their presence in the parasite's gut $^{[21,22]}$ and might thus be intimately linked to its ability to uptake nutrients.

Although the detailed mechanisms of SAPLIP-mediated enzyme activation are unknown, it is generally assumed that these proteins remodel the membrane by interacting with lipids; they are also able to interact directly with lipid degrading enzymes, thus providing a platform for lipid hydrolases. ${ }^{[23]}$ SAPLIPs are crucial molecules for the survival of many organisms. For instance, SAPLIP-deficient Caenorhabditis elegans is overcome by bacteria in culture, ${ }^{[2]}$ because the worm is unable to induce bacterial lysis. In humans, deficiencies in saposins A, B or C lead to Krabbe-like disease, ${ }^{[25]}$ metachromatic leukodystropy ${ }^{[26]}$ and Gaucher-like disease, ${ }^{[27]}$ respectively.

SAPLIPs are also important contributors in host interactions. Amoebapores A-C are SAPLIPs and virulence factors of the agent of amoebic dysentery, Entamoeba histolytica, a protozoan parasite that kills host cells by forming pores in their lipid membranes. ${ }^{[28]}$ In addition, SAPLIPs from the metazoan liver flukes Clonorchis sinensis (clonorin) ${ }^{[29]}$ and Fasciola hepatica (Fh-SAP-2) ${ }^{[30]}$ are haemolytic and are likely intimately involved in host responses. Thus, in addition to their diagnostic potential, ${ }^{[31,32]}$ they are immunogenic. For instance, a recombinant form of Fh-SAP-2 has achieved $>80 \%$ protection against $F$. hepatica challenge infection in rabbits ${ }^{[30]}$ and a reduction in worm burdens in mice when vaccinated with a cDNA construct of Fh-SAP-2. ${ }^{[33]}$ This protection level compares with other vaccine targets for fascioliasis with efficacies of $40-74 \% .{ }^{[34-36]}$ Moreover, sera 
from experimental mice infected with $S$. mansoni reacted to Fh-SLP-2 in vitro, thus showing crossreactivity ${ }^{[37]}$ and suggesting that helminth SAPLIPs might be able to protect against trematodes more broadly.

Interestingly, a number of human Sap domain-containing proteins, including granulysin, NK-lysin and surfactant protein $\mathrm{B}$, have activity against a range of targets, such as tumor cells, bacteria, fungi and parasites, ${ }^{[38-40]}$ and, accordingly, are considered components of the innate (not antibody provided) immune system.

Despite the conservation of the Sap domain, the fold of individual SAPLIPs is adapted to carry out particular and diverse functions at biological membranes ranging from cytolytic properties of amoebapores, ${ }^{[28]}$ granulysin $^{[41]}$ and NK-lysin ${ }^{[42]}$ to plant aspartic proteases ${ }^{[43]}$ and neurotrophic factors. ${ }^{[44]}$ Due to the many variations and adaptations in protein families such as this, experimental three-dimensional structures of individual members are indispensable.

Typical structural characteristics of the Sap domain include a jaw- like arrangement of a four- or five-helix bundle where a kink in the third helix can lead to occurrence of two helices (see Figure 1). Three conserved dithioether bonds link the helices within each lobe but not across the jaw.

Recently, we determined the crystal structures of two novel SAPLIPs (called Na-SLP-1 and AcSLP-1) from human and dog hookworms Necator americanus and Ancylostoma caninum. ${ }^{[4]}$ Despite the same numbering, these two proteins are not close orthologues - a conclusion that is supported by insights gained into the molecular mechanisms of these proteins. Although the crystal structures of both $\mathrm{Na}$-SLP-1 and Ac-SLP-1 suggest that these molecules form differing P2symmetric dimers, only Ac-SLP-1 exists as dimers in solution. Based on the observed threedimensional Ac-SLP-1 dimer, it has been possible to suggest a likely membrane-associated mechanism (see below), which would not have been possible without knowledge obtained from the crystal structure.

A particular feature of many Sap domains is the loose packing of side chains in the core, where large void volumes result in an 'oily' and mobile core. ${ }^{[41]}$ In the three-dimensional crystal structure of $\mathrm{Na}$-SLP-1, at least a dozen void volumes $\left(>5 \AA^{3}\right.$ ) have been found, the largest one being $43 \AA^{3}$. In contrast, $A c$-SLP-1 presents itself as a well-ordered protein, from which void volumes are absent and, therefore, devoid of an 'oily' feature.

The membrane-associated activities of Sap domain containing proteins appear to be quite varied (see Figure 2). The main characteristics identified to date are described in the following:

(1) Extensive protein-protein interactions have been observed in the crystal structure of human granulysin, ${ }^{[28]}$ suggesting that a two-dimensional layer of granulysin covers the membrane surface, achieved by a roll-on mechanism. The lipid extraction activity has been proposed to occur via a scissor-like action of the two 'jaws'. In contrast to amoebapore A (see below), the membrane permeabilising species of granulysin and NK-lysin appear to be monomeric within a membraneinfesting carpet. In this model, each monomer is bound to the membrane surface via a highly positively charged surface area, and the high density of positive charges on the SAPLIPs is assumed to cause membrane destabilisation by electroporation. ${ }^{[46]}$

Noteworthy is the observation that the crystal structure of Na-SLP-1 from human hookworm and the membrane binding data obtained for this protein ${ }^{[44]}$ is similar to granulysin; however, neither haemolytic nor liposome destabilisation has been observed thus far for Na-SLP-1.

(2) For human saposins C and D, a mechanism has been proposed that comprises the annealing of dimeric species to the membrane via the amphipathic helices $\alpha 2$ and $\alpha 3-4$, respectively. This 
generates conformations competent for lipid extraction ${ }^{[47]}$ and enables the saposins to function as activators that deliver lipids to lipases which are unable to penetrate the membrane.

(3) A clip-on model has been suggested for membrane fusion events mediated by saposin C. Again, the occurrence of a saposin dimer species is required for this membrane-linked mechanism to proceed. ${ }^{[48]}$

(4) The crystal structure of Ac-SLP-1, a novel SAPLIP from the dog hookworm, reveals a symmetrical dimer and a central, buried hydrophobic surface that mainly comprises the solventexposed side of helix $\alpha 3{ }^{[45]}$ Supported by native gel and size exclusion chromatography data, ${ }^{[45]}$ this dimer is believed to be the physiologically relevant species of molecule in solution. The dimer features a linear groove, lined with acidic residues on one side of the globular shape, connecting large cavities at the distal ends. Although the physiological role of these connected cavities is not yet known, its structural features are poised to shuttle molecules between the membrane surface and the hydrophilic medium beneath it. Intriguingly, the crystal structure shows a citrate and two HEPES molecules (from the crystallisation buffer) bound in the connecting groove. Since the AcSLP-1 crystal structure does not display any obvious, privileged sites for membrane interactions, a membrane binding mechanism that involves disassembly of the dimer upon initial contact with the membrane surface and annealing of the hydrophobic side of helix $\alpha 3$ to the membrane seems plausible.

(5) The pore-forming amoebapore A from the parasitic amoeba E. histolytica has been shown in an NMR study to assemble into dimers in a pH-dependent fashion, mediated by the single histidine residue (His75) of this protein. ${ }^{[28]}$ By means of molecular modelling, a trimer of dimers has been proposed that has a mainly hydrophobic outer torus surface, whereas the surface lining the putative pore shows mainly basic character. The modelled pore diameter of about $20 \AA$ is in excellent agreement with its pore size estimated by electrophysiological means. ${ }^{[49]}$

(6) The crystal structure of saposin B revealed two dimers, a symmetric (crystallographic) dimer, and an asymmetric dimer with a large hydrophobic pocket of about $900 \AA^{3}$ at the subunit interface that can harbour lipid molecules. ${ }^{[50]}$ Based on these findings, it has been proposed that a conformational switch governing flexibility at the opening of the hydrophobic cavity facilitates the transition between the active and inactive form of this protein. When active, saposin B forms a complex with extracted lipid molecules that is recognisable by lipases and hence, facilitates membrane degradation. ${ }^{[50]}$ While the precise mode of saposin B membrane association has yet to be proposed, the use of a conformationally flexible dimer for lipid extraction suggests that the pathway may be similar to those of saposins $\mathrm{C}$ and $\mathrm{D}$.

\section{Excreted/secreted products: SCP/TAPS proteins}

Owing to their life style, parasitic worms release a myriad of factors in order to regulate the immune response of the host. ${ }^{[51]}$ The decoding of this secretome (= exported proteome) has experienced a recent boost with the deployment of modern genomic and transcriptomic sequencing ${ }^{[52]}$ as well as proteomic analyses. ${ }^{[53]}$ Since nematode secretomes are complex in composition, it is not a trivial task to identify biologically active components. Two main approaches are currently being applied: (i) a systematic analysis that attempts to identify secreted proteins that are conserved among inhabitants of a particular ecological niche (such as for example the gastrointestinal tract, bile duct, etc), and (ii) a pragmatic approach that identifies the most abundant 
proteins secreted within the host environment.

The two approaches may not need to be exclusive. The activation-associated secreted proteins (ASPs) of hookworms, for example, are amongst the ten most abundant groups of proteins in hookworms, ${ }^{[54]}$ and their transcription is highly up-regulated when the free-living parasite transforms to the parasitic stages. ${ }^{[55]}$ ASPs belong to the family of sperm-coating protein (SCP)-like extracellular proteins, also called SCP/TAPS proteins, and as such are part of the cysteine-rich secretory protein (CRISP) superfamily. The involvement of SCP/TAPS proteins in fundamental biological processes in eukaryotes is undoubted, and there are prominent examples of highly bioactive molecules, such as the allergens from the venoms of fire ants (Sol i2, Sol i3), wasps (Ves V5), snakes (e.g. triflin) and others. ${ }^{[54]}$ In $S$. mansoni, for example, members of this protein family are known as the Venom Allergen-Like proteins (Sm-VALs).

ASPs, ${ }^{[56]}$ including Sm-VALs, ${ }^{[57]}$ have received attention as targets for anti-parasite intervention, since they are expressed abundantly in the infective stages of the parasites and are also immunogenic. With the help of structural biology methodologies, we have recently embarked on systematic analyses of ASPs, and developed a framework of classification for these proteins using structure-based amino acid alignments ${ }^{[58,59]}$ and phylogeny, ${ }^{[54,60-62]}$ yielding (at least) three different groups.

Interestingly, ASPs often share low amino acid sequence identities among one another, but possess a conserved fold in the N-terminal PR (or CAP) domain, characterised by a central anti-parallel $\beta$ sheet that is embraced by helical motifs ( $\alpha-\beta-\alpha$ sandwich), and several disulphide bonds that provide structural stability of this fold (see Figure 3). The fold of the C-terminal domain of SCP/TAPS proteins can vary, but in the case of hookworm ASPs, Hinge-like sequence of CRISPs with a conserved cystein pattern of $\mathrm{C}-\mathrm{X}_{5}-\mathrm{C}-\mathrm{X}_{7}-\mathrm{C}-\mathrm{X}_{4}-\mathrm{C}^{[63]}$ have been found. ${ }^{[59]}$ The three-dimensional structures of representative members of Group 1 ( $\mathrm{Na}$-ASP- $2^{[64]}$ ) and Group 2 (Ac-ASP-7 ${ }^{[59]}$ ) ASPs confirm the significant structural differences between these groups, and crystallographic analyses have also revealed the presence of metal-binding sites that differ between the two groups. For Group 1 ASPs, one of the prominent structural features is an equatorial groove that extends approximately around half of the molecule and harbours a tandem histidine motif (His88 and His148 in Na-ASP-2) which has the ability to bind a variety of transition metals. ${ }^{[65]}$ In Group 2 ASPs, this structural feature is absent.

That ASPs are secreted proteins suggests particular roles at the parasite-host interface, including: (i) decoy molecules to divert the immunological response by the host; (ii) modulation/suppression of the immunological response by interaction with relevant host receptors; and (iii) protection of the parasite by removal of undesired chemical entities. Hypothetically, using the metal binding sites observed in Group 1 and Group 2 ASPs, ${ }^{[59,}{ }^{65]}$ these proteins could act as catcher molecules for undesired metal ions that might be damaging to the parasite.

However, the presence of the equatorial groove on Group 1 ASPs stimulated the hypothesis that peptides might be bound in this location. ${ }^{[59]}$ By screening peptide libraries by phage panning, a peptide sequence has recently been identified that specifically binds to Na-ASP-2 and shows high identity to a peptide of the extracellular domain of the human SK3 channel. ${ }^{[64]}$ Direct interactions between an SK3 peptide and Na-ASP-2 could be confirmed by modelling and thermostability experiments. ${ }^{[65]}$

Although ion channels are best known for their roles in neuronal and cardiac action potentials, their importance in immune cells is less well characterised. Recent reports indicate that SK channels play a role in mediating reactive oxygen species (ROS) production. ${ }^{[66]}$ Putatively, ASPs may act similarly to the bee venom apamin and block the production of $\operatorname{ROS}^{[66]}$ that are toxic to invading hookworm larvae as they migrate through host tissues to the gastrointestinal tract. 
To date, there is only one hookworm ASPs for which a molecular biological function has been experimentally confirmed. Neutrophil inhibitory factor (Ac-NIF) is a Group 1 ASP from A. caninum and modulates the immune response by inhibiting neutrophil recruitment by direct interaction with complement receptor $3 .{ }^{[67]}$ Intriguingly, Ac-NIF has recently been shown in a mouse model to inhibit early stages of diabetic retinopathy without compromising the immune surveillance. ${ }^{[68]}$ Less is known for another A. caninum ASP termed hookworm platelet inhibitor (HPI), which acts as an antagonistic ligand of cell surface integrins $\left(\alpha_{\mathrm{IIb}} \beta_{3}\right.$ and $\left.\alpha_{2} \beta_{1}\right)$, resulting in the inhibition of the aggregation and adhesion of platelets. ${ }^{[69]}$

To date, the interaction of Ac-NIF with complement receptor 3 constitutes the only experimentally proven interaction of an ASP with a human receptor. As a member of the complement system, complement receptor 3 (a heterodimeric complex consisting of $\alpha_{\mathrm{M}^{-}}$and $\beta 2$-integrin) is a cell surface receptor on human leukocytes and macrophages that constitutes the innate immune system and helps to clear pathogens. Integrins consist of a family of proteins that form heterodimers with one $\alpha$ and one $\beta$ chain. These heterodimers are the major adhesion receptors and mediate essential cellular functions. They play a role in cell-cell interaction, development, immune responses, leukocyte traffic and are at the heart of many diseases including cancer ${ }^{[70]}$ The human genome comprise of 18 $\alpha$ subunits and $8 \beta$ subunits that assemble into 24 distinct integrins with specific, non-redundant function. Whereas $\beta_{2}$-integrins have been implicated in the recognition and binding of molecules originating from bacterial and yeast pathogens, viruses appear to use mainly $\beta_{3}$-integrins (and to a lesser extent $\beta_{1}, \beta_{5}$ or $\beta_{7}$-integrins) for cell entry. ${ }^{[71]}$ Intriguingly, $A c$-NIF appears to specifically bind to the $\alpha$ subunit of complement receptor $3^{[72]}$ and localise to the I-domain of $\alpha_{M}{ }^{[73]}$ that contains the metal ion-dependent adhesion site (MIDAS). ${ }^{[74]}$ In terms of structural elucidation, the molecular interactions between viruses or viral proteins with integrins have been investigated in many instances, and include three-dimensional structures by electron microscopy or crystallography. ${ }^{[71]}$ Surprisingly, the investigation of parasite interactions with integrins is much less advanced (see Table 1).

\section{Future directions}

\section{Saposin-like proteins}

As potentially membrane-lytic proteins, SAPLIPs are likely to play key roles in schistosome metabolism; they are abundant, with 14 Sap-domain proteins known for S. japonicum. ${ }^{[22]}$ The molecules are also highly conserved across the three main species of Schistosoma (S. mansoni, S. japonicum and $S$. haematobium) which cause the majority of cases of schistosomaisis in humans, yet they share little amino acid sequence identity to the saposins from the human host. This information indicates that this group of molecules will be interesting to explore biologically in schistosomes and other blood-feeding helminths and that they have potential as targets of parasite intervention. The genomes of all three human schistosomes have been sequenced, and all three trematodes can be maintained in the laboratory in rodents and snails. Both larval and adult worms of schistosomes can be maintained in culture in vitro and are amenable to RNA interference (RNAi). ${ }^{[72]}$ In contrast to a number of other parasites, where RNAi has not been reported or inconsistent susceptibilities have been observed, ${ }^{[73]}$ schistosomes, particularly $S$. mansoni, should allow integrated studies of SAPLIPs, combining functional explorations with elucidation of these proteins at the molecular, structural and/or immuno-biochemical levels. Conclusions regarding homologous structure-function relationships, from one individual SAPLIP to another, are not advisable for the reasons discussed in this review. However, there is an anticipation that comparison of SAPLIP family members, and particularly orthologous proteins, across different nematode species - after phylogenies have been established - should allow conclusions to be made regarding 
shared SAPLIP mechanisms among species. Ultimately, this might provide a basis for the definition of intervention targets and, subsequently, the design of treatments with relatively broad applicability.

\section{SCP/TAPS proteins}

Although SCP/TAPS proteins (including ASPs) appear to be central to the interplay between parasite and host, there are very scarce data about the identity of the host targets and very little structural information that could provide clues about molecular mechanisms, thus impacting on the opportunities for rational/structure-guided discovery of novel interventions.

An understanding of the involvement of structural motifs of integrins in this context is just beginning to emerge. On the one hand, host integrins might be attacked by proteins secreted by parasite, to interfere with signalling and promotion of cell-cell/cell-ECM contacts, ultimately modulating immune responses. Interestingly, integrin-like structural features have been observed in parasite surface proteins (e.g. TRAPs) and may thus provide pathways of parasite entry by forming multivalent adhesive junctions. Information about three-dimensional structures of such interactions are thus in high demand, particularly because comparative modelling may not allow the extrapolation of findings obtained from "prototype" complexes, as subtle differences even among members of the same protein fold can lead to different interactions. Mechanisms of conformational regulation, such as subtle differences in the metal coordination of the metal ion-dependent adhesion site (MIDAS) of $\beta 2$-integrin regulating integrin function ${ }^{[77]}$ makes the requirement for atomic details of the particular complexes even more important.

Integrins are known targets of therapeutic drugs against thrombosis and inflammation ${ }^{[78]}$ and thus might have potential as drug targets in parasites. We anticipate that future crystal structure studies of integrin-ASP complexes will lead to discoveries that can assist the development of novel therapeutic approaches for infectious and inflammatory diseases.

\section{Conclusions}

As with many other areas of the life sciences, structural biological investigations by X-ray crystallography have contributed immensely to our understanding of pathogen-host interactions. The genomic and transcriptomic data that are increasingly becoming available from parasitic organisms provide an unprecedented resource for targeted investigation of parasite-host protein complexes. Such crystal structures are in high demand, as the molecular armory of parasites is manifold, and comparative modelling will not be able to account for the significant differences in the structure-function relationships. The protein families, saposin-like proteins and SCP/TAPS proteins, highlighted in this review are key examples in this context, since both families are characterised by highly conserved three-dimensional folds, but structure-function relationships can substantially differ among the individual members, owing to the low amino acid sequence identity between or among them. Protein crystallography will thus remain an integral part in establishing the molecular mechanisms of infectious diseases as well as the development of novel interventions in this area.

\section{Acknowledgements}

AH's research is funded by the National Health and Medical Research Council (NHMRC), the Australian Research Council (ARC) and the Rebecca L Cooper Medical Research Foundation. RBG's research is funded mainly through the ARC, NHMRC, Melbourne Water Corporation and Yourgene Bioscience, and supported by a Victoria Life Sciences Computation Initiative (VLSCI; 
grant number VR0007) on its Peak Computing Facility at the University of Melbourne, an initiative of the Victorian Government. MKJ's research is supported by NHMRC, ARC and the Peter and Mary Stone Foundation.

\section{Author contributions}

$\mathrm{AH}$ wrote the paper with critical input from all authors. 


\section{References}

[1] S. Kutz, A. Dobson, E. Hoberg, Science 2009, 326, 1187-1188.

[2] J. N. Mills, K. L. Gage, A. S. Khan, Environ. Health Perspect. 2010, 118, 1507.

[3] C. E. Barry, Curr. Top. Med. Chem. 2011, 11, 1216.

[4] C. Arama, M. Troye-Blomberg, J. Intern. Med. 2014, 275, 456.

[5] K. J. Evans, L. Kedzierski, J. Trop. Med. 2012, 2012, 892817.

[6] C. Y. Leow, C. Willis, A. Osman, L. Mason, A. Simon, B. J. Smith, R. B. Gasser, M. K. Jones, A. Hofmann, FEBS J. 2013, 281, 1209-1225.

[7] A. Winter, A. M. Yusof, E. Gao, H. L. Yan, A. Hofmann, FEBS J. 2006, 273, 3238.

[8] S. K. Weeratunga, A. Osman, N. J. Hu, C. K. Wang, L. Mason, S. Svärd, G. Hope, M. K. Jones, A. Hofmann, J. Mol. Biol. 2012, 423, 169.

[9] A. Hofmann, A. Osman, C. Y. Leow, P. Driguez, D. P. McManus, M. K. Jones, BioEssays 2010, 32, 967.

[10] C. Y. Leow, C. Willis, R. B. Gasser, A. Hofmann, M. K. Jones, Br. J. Pharmacol. 2014, in press.

[11] L. Schulte, E. Lovas, K. Green, G. N. Gobert, J. Mulvenna, G. Morgan, M. K. Jones, Int. J. Parasitol. 2013, 43, 785.

[12] E. Hanssen, C. Dekiwadia, D. T. Riglar, M. Rug, L. Lemgruber, A. F. Cowman, M. Cyrklaff, M. Kudryashev, F. Frischknecht, J. Baum et al., Cell Microbiol. 2013, 15, 1457.

[13] C. Cantacessi, A. Hofmann, B. E. Campbell, R. B. Gasser, In: Molecular diagnostics and highthroughput strategies in veterinary infection biology 2014 (Springer), in press.

[14] P. Sarnpitak, P. Mujumdar, P. Taylor, M. Cross, D. Gorse, D. Camp, M. Krasavin, A. Hofmann, submitted 2014.

[15] A. Hofmann, C. K. Wang, A. Osman, D. Camp, Struct. Chem. 2010, 21, 1117.

[16] K. Sandhoff, T. Kolter, Trends Cell. Biol. 1996, 6, 98.

[17] T. Linke, G. Wilkening, F. Sadeghlar, H. Mozcall, K. Bernardo, E. Schuchman, K. Sandhoff, J. Biol. Chem. 2001, 276, 5760.

[18] W. Yuan, X. Qi, P. Tsang, S. Kang, P. A. Illarionov, G. S. Besra, J. Gumperz, P. Cresswell, Proc. Natl. Acad. Sci. U S A 2007, 104, 5551.

[19] F. Ciaffoni, R. Salvioli, M. Tatti, G. Arancia, P. Crateri, A. M. Vaccaro, J. Biol. Chem. 2001, 276, 31583.

[20] T. A. Don, Y. Oksov, S. Lustigman, A. Loukas, Parasitology 2007, 134, 427.

[21] S. Nawaratna, D. McManus, L. Moertel, G. Gobert, M. Jones, PLoS Negl. Trop. Dis. 2011, 5, e1043.

[22] J. Chen, T. Zhang, C. Ju, B. Xu, Y. Lu, X. Mo, S. Chen, Y. Fan, W. Hu, X. Zhou, J. Proteom. 2014, 98, 289.

[23] G. Wilkening, T. Linke, K. Sandhoff, J. Biol. Chem. 1998, 273, 30271.

[24] T. Roeder, M. Stanisak, C. Gelhaus, I. Bruchhaus, J. Grötzinger, M. Leippe, Dev. Comp. Immunol. 2010, 34, 203.

[25] J. Matsuda, M. T. Vanier, Y. Saito, J. Tohyama, K. Suzuki, K. Suzuki, Hum. Mol. Genet. 2001, $10,1191$.

[26] K. A. Kretz, G. S. Carson, S. Morimoto, Y. Kishimoto, A. L. Fluharty, J. S. O'Brien, Proc. Natl. Acad. Sci. U S A 1990, 87, 2541.

[27] Y. Sun, B. Liou, H. Ran, M. R. Skelton, M. T. Williams, C. V. Vorhees, K. Kitatani, Y. A. Hannun, D. P. Witte, Y. Xu et al., Hum. Mol. Genet. 2010, 19, 1088.

[28] O. Hecht, N. A. Van Nuland, K. Schleinkofer, A. J. Dingley, H. Bruhn, M. Leippe, J. Grötzinger, J. Biol. Chem. 2004, 279, 17834.

[29] J. Lee, P. Cho, T. Y. Kim, S. Kang, K. Song, S. Hong, Biochem. Biophys. Res. Commun. 2002, 
296, 1238.

[30] A. M. Espino, G. V. Hillyer, J. Parasitol. 2004, 90, 876-879.

[31] J. Lee, T. Kim, X. Gan, S. Kang, S. Hong, Parasitol Int. 2003, 52, 175.

[32] O. Figueroa-Santiago, B. Delgado, A. M. Espino, Diagn. Microbiol. Infect. Dis. 2011, 70, 355.

[33] A. M. Espino, A. Morales, B. Delgado, F. M. Rivera, O. Figueroa, E. Suárez, Ethn. Dis. 2010, 20, S1-17-23.

[34] C. A. Morrison, T. Colin, J. L. Sexton, F. Bowen, J. Wicker, T. Friedel, T. W. Spithill, Vaccine 1996, 14, 1603.

[35] J. P. Dalton, S. McGonigle, T. P. Rolph, S. J. Andrews, Infect. Immun. 1996, 64, 5066.

[36] A. Muro, V. Ramajo, J. López, F. Simón, G. V. Hillyer, Vet. Parasitol. 1997, 69, 219.

[37] D. Torres, A. M. Espino, Infect. Immun. 2006, 74, 4932.

[38] D. Andreu, C. Carreño, C. Linde, H. G. Boman, M. Andersson, Biochem. J. 1999, 344 Pt 3, 845.

[39] Z. Wang, E. Choice, A. Kaspar, D. Hanson, S. Okada, S. C. Lyu, A. M. Krensky, C. Clayberger, J. Immunol. 2000, 165, 1486.

[40] L. Yang, J. Johansson, R. Ridsdale, H. Willander, M. Fitzen, H. T. Akinbi, T. E. Weaver, J. Immunol. 2010, 184, 975.

[41] D. H. Anderson, M. R. Sawaya, D. Cascio, W. Ernst, R. Modlin, A. Krensky, D. Eisenberg, J. Mol. Biol. 2003, 325, 355.

[42] E. Liepinsh, M. Andersson, J. M. Ruysschaert, G. Otting, Nat. Struct. Biol. 1997, 4, 793.

[43] J. Kervinen, G. J. Tobin, J. Costa, D. S. Waugh, A. Wlodawer, A. Zdanov, EMBO J. 1999, 18, 3947.

[44] B. C. Bornhauser, P. Olsson, D. Lindholm, J. Biol. Chem. 2003, 278, 35412.

[45] C. Willis, C. Wang, A. Osman, A. Simon, D. Pickering, J. Mulvenna, A. Riboldi-Tunicliffe, M. Jones, A. Loukas, A. Hofmann, PLoS ONE 2011, 6, e25369.

[46] M. Miteva, M. Andersson, A. Karshikoff, G. Otting, FEBS Lett. 1999, 462, 155.

[47] M. Rossmann, R. Shultz-Heienbrok, J. Behlke, N. Remmel, C. Alings, K. Sandhoff, W. Saenger, T. Maier, Structure 2008, 16, 809.

[48] Y. Wang, G. A. Grabowski, X. Qi, Arch. Biochem. Biophys. 2003, 415, 43.

[49] T. Gutsmann, B. Riekens, H. Bruhn, A. Wiese, U. Seydel, M. Leippe, Biochemistry 2003, 42, 9804.

[50] V. Ahn, K. Faull, J. Whitelegge, A. Fluharty, G. Prive, Proc. Natl. Acad. Sci. USA 2003, 100, 38.

[51] J. P. Hewitson, J. R. Grainger, R. M. Maizels, Mol. Biochem. Parasitol. 2009, 167, 1.

[52] D. C. Koboldt, K. M. Steinberg, D. E. Larson, R. K. Wilson, E. R. Mardis, Cell 2013, 55, 27.

[53] J. Mulvenna, B. Hamilton, S. H. Nagaraj, D. Smyth, A. Loukas, J. J. Gorman, Mol. Cell. Proteomics 2009, 8, 109.

[54] C. Cantacessi, B. E. Campbell, A. Visser, P. Geldhof, M. J. Nolan, A. J. Nisbet, J. B. Matthews, A. Loukas, A. Hofmann, D. Otranto et al., Biotechnol. Adv. 2009, 27, 376.

[55] B. J. D. Datu, R. B. Gasser, S. H. Nagaraj, E. K. Ong, P. O'Donoghue, R. McInnes, S. Ranganathan, A. Loukas, PLoS Negl. Trop. Dis. 2008, 2, e130.

[56] Loukas A., Bethony J., Brooker S. \& Hotez P., Lancet Infect Dis 2006, 6, 733-741.

[57] L. P. Farias, D. Rodrigues, V. Cunna, H. K. Rofatto, E. L. Faquim-Mauro, L. C. C. Leite, PLoS Negl. Trop. Dis. 2012, 6, e1510.

[58] C. K. Wang, U. Broder, S. K. Weeratunga, R. B. Gasser, A. Loukas, A. Hofmann, Bioinformatics 2012, 28, 1026.

[59] A. Osman, C. K. Wang, A. Winter, A. Loukas, L. Tribolet, R. B. Gasser, A. Hofmann, 
Biotechnol Adv. 2012, 30, 652.

[60] C. Cantacessi, A. Hofmann, N. D. Young, R. S. Hall, A. Loukas, R. B. Gasser, PLoS ONE 2012, 7, e31164.

[61] C. Cantacessi, J. Mulvenna, N. D. Young, M. Kasny, P. Horak, A. Aziz, A. Hofmann, A. Loukas, R. B. Gasser, Mol. Cell. Proteomics 2012, 11, 1340.

[62] E. M. Schwarz, P. K. Korhonen, B. E. Campbell, N. D. Young, A. R. Jex, A. Jabbar, R. S. Hall, A. Mondal, A. C. Howe, J. Pell et al., Genome Biol. 2013, 14, R89.

[63] G. M. Gibbs, K. Roelants, M. K. O'Bryan, Endocr. Rev. 2008, 29, 865.

[64] O. A. Asojo, G. Goud, K. Dhar, A. Loukas, B. Zhan, V. Deumic, S. Liu, G. E. O. Borgstahl, P. J. Hotez, J. Mol. Biol. 2005, 346, 801.

[65] L. Mason, L. Tribolet, A. Simon, N. von Gnielinski, L. Nienaber, P. Taylor, C. Willis, M. K. Jones, P. W. Sternberg, R. B. Gasser et al., Int. J. Biochem. Cell Biol. 2014, 50, 146.

[66] A. J. Fay, X. Qian, Y. N. Jan, L. Y. Jan, Proc. Natl. Acad. Sci. U S A 2006, 103, 17548.

[67] P. J. Muchowski, L. Zhang, E. R. Chang, H. R. Soule, E. F. Plow, M. Moyle, J. Biol. Chem. 1994, 269, 26419.

[68] A. A. Veenstra, J. Tang, T. S. Kern, PLoS One 2013, 8, e78405.

[69] A. Del Valle, B. F. Jones, L. M. Harrison, R. C. Chadderdon, M. Cappello, Mol. Biochem. Parasitol. 2003, 129, 167.

[70] R. O. Hynes, Cell 2002, 110, 673.

[71] P. L. Stewart, G. R. Nemerow, Trends Microbiol. 2007, 15, 500.

[72] S. Stefanic, J. Dvorak, M. Horn, S. Braschi, D. Sojka, D. S. Ruelas, B. Suzuki, K. Lim, S. D. Hopkins, J. H. McKerrow et al., PLoS Negl. Trop. Dis. 2010, 4, e850.

[73] A. G. Maule, P. McVeigh, J. J. Dalzell, L. Atkinson, A. Mousley, N. J. Marks, Trends Parasitol. 2011, 27, 505.

[74] M. Moyle, D. L. Foster, D. E. McGrath, S. M. Brown, Y. Laroche, J. De Meutter, P. Stanssens, C. A. Bogowitz, V. A. Fried, J. A. Ely et al., J. Biol. Chem. 1994, 269, 10008.

[75] L. Zhang, E. F. Plow, J. Biol. Chem. 1997, 272, 17558.

[76] J. O. Lee, P. Rieu, M. A. Arnaout, R. Liddington, Cell 1995, 80, 631.

[77] B. Mahalingam, K. Ajroud, J. L. Alonso, S. Anand, B. D. Adair, A. L. Horenstein, F. Malavasi, J. Xiong, M. A. Arnaout, J. Immunol. 2011, 187, 6393.

[78] S. L. Goodman, M. Picard, Trends Pharmacol. Sci. 2012, 33, 405.

[79] J. Vazquez-Prado, I. Meza, Arch. Med. Res. 1992, 23, 125.

[80] P. Talamas-Rohana, J. L. Rosales-Encina, M. C. Gutierrez, V. Hernandez, Arch. Med. Res. 1992, 23, 119.

[81] G. N. Sarma, V. A. Manning, L. M. Ciuffetti, P. A. Karplus, Plant Cell 2005, 17, 3190.

[82] J. M. Harper, E. F. Hoff, V. B. Carruthers, Mol. Biochem. Parasitol. 2004, 134, 201.

[83] S. Gantt, C. Persson, K. Rose, A. J. Birkett, R. Abagyan, V. Nussenzweig, Infect. Immun. 2000, 68, 3667.

[84] K. J. Robson, S. Naitza, G. Barker, R. E. Sinden, A. Crisanti, Mol. Biochem. Parasitol. 1997, $84,1$.

[85] R. R. Akhouri, A. Sharma, P. Malhotra, A. Sharma, Malaria J. 2008, 7, 63.

[86] Y. Cho, B. F. Jones, J. J. Vermeire, L. Leng, L. DiFedele, L. Harrisson, H. Xiong, Y. Kwong, Y. Chen, R. Bucala et al., J. Biol. Chem. 2007, 282, 23447.

[87] Y. Cho, J. J. Vermeire, J. S. Merkel, L. Leng, X. Du, R. Bucala, M. Cappello, E. Lolis, Chem. Biol. 2011, 18, 1089.

[88] B. Zhan, H. Santiago, B. Keegan, P. Gillespie, J. Xue, J. Bethony, L. M. de Oliveira, D. Jiang, D. Diemert, S. Xiao et al., Parasite Immunol. 2012, 34, 404.

[89] L. Tribolet, C. Cantacessi, D. A. Pickering, S. Navarro, D. L. Doolan, A. Trieu, H. Fei, Y. 
Chao, A. Hofmann, R. B. Gasser, P. R. Giacomin, Alex Loukas, submitted 2014.

[90] L. Kedzierski, J. Montgomery, D. Bullen, J. Curtis, E. Gardiner, A. Jimenez-Ruiz, E. Handman, J. Immunol. 2004, 172, 4902.

[91] M. Kelleher, S. F. Moody, P. Mirabile, A. H. Osborn, A. Bacic, E. Handman, Infect. Immun. 1995, 63, 43.

[92] L. Chen, K. V. N. Rao, Y. He, K. Ramaswamy, J. Biol. Chem. 2002, 277, 34329.

[93] F. Trottein, S. Nutten, J. P. Papin, C. Leportier, O. Poulain-Godefroy, A. Capron, M. Capron, J. Immunol. 1997, 159, 804.

[94] W. DeLano, http://www.pymol.org 2002. 


\section{Table 1}

A non-exhaustive survey of molecular parasite-host interactions employing cell surface receptors

\section{Parasite protein}

\section{Functional role}

Entamoeba histolytica

$37 \mathrm{kDa}$ surface protein Unknown

$140 \mathrm{kDa}$ surface protein Unknown

Pyrenophora triticirepentis

ToxA

Secreted integrin

antagonist with RGD

motif

Toxoplasma gondii, Plasmodium

TRAP

Hepatocyte invasion

Sulfogalactosy l-cerebrosides (sulfatides),

$\beta_{2}$-integrin,

SH3-domain

containing

proteins

Ancylostoma ceylanicum

Ace-MIF

Cytokine ortholog

CD74

Ancylostoma caninum

NIF
Heparin?,

TRAP proteins possess multiple adhesion domains, including an A-

Structures of the human thrombospondin-1 type 1 repeats (PDB accession code 1lsl) and the TSR domain of a Plasmodium TRAP (2bbx) are known.

\section{Reference} PDB accession codes: 1zld, 1ale).

The macrophage migration inhibiting factor (MIF) acts as a lymphocyte chemo-attractant, similar to the human orthologue and possesses tautomerase activity. Vaccination provided partial protection from disease. Crystal structures of the protein in the apo (PDB accession code: 2os5) and drug-bound states are known (3rf4, 3rf5).

Complement Interaction with complement receptor 3 and inhibition of neutrophil ${ }^{[67,68]}$ 
Necator americanus

Na-ASP-2

Unknown

\section{Leishmania}

Parasite surface antigen

2 (PSA-2)

Lipophosphoglycan

\section{Schistosoma mansoni}

$23 \mathrm{kDa}$ secreted protein Host cell apoptosis

Integrin-like cell surface receptors
Modulation of host phagocytosis and chemotaxis
$\alpha_{\mathrm{II}} \beta_{3}$ integrin, Blocks platelet aggregation and adhesion.

SK3 channel? A vaccine candidate that suffers from IgE-mediated allergic reactions in humans. Crystal structures of apo and metal-bound $\mathrm{Na}$-ASP-2 are known (PDB accession codes: 1u534, 4nui, 4nuk, 4nun, 4nuo).

Complement Strong indications that the LRR domain binds the CR3 and contributes to receptor 3 invasion.

$\left(\alpha_{\mathrm{M}} \beta_{2^{-}}\right.$

integrin)

Complement receptor 3

$\left(\alpha_{\mathrm{M}} \beta 2-\right.$

integrin)

Direct interactions with lipopolysaccharide-binding site of CR3; opsonisation of C3b and iC3b.

Death

receptors

Host cell apoptosis is a common strategy among parasites, including schistosomes, N. americanus, F. hepatica, Paragonimus westermani, Taenia crassiceps, Trypanosoma cruzi, Leishmania donovani, and Cryptosporidium parvum.

CD15 Parasite CR3-like proteins cause antibody-dependent cell-mediated cytotoxicity (ADCC) of macrophages. 


\section{Figure Legends}

\section{Figure 1}

Crystal structures of Na-SLP-1 (PDB accession code 3s63) and Ac-SLP-1 (PDB accession code 3s64). The topology of the Sap domain fold is highlighted by the blue/orange colouring. Disulphide linkages are rendered as stick models and coloured yellow. Both structures reveal dimeric arrangements with interface areas of $571 \AA^{2}$ and $767 \AA^{2}$, respectively. As suggested by the rather small interface area observed in the $N a$-SLP-1 crystal structure, this protein exists exclusively as monomer in solution. Ac-SLP-1, in contrast, is dimeric in solution. The dimer interface of Ac-SLP1 features a long groove in which a citrate and two HEPES molecules were found in P2-symmetric positions. The citrate molecule occupies a special position and only one conformation is shown for clarity. Protein structures were rendered with PyMOL. ${ }^{[94]}$

\section{Figure 2}

Versatile membrane interactions by Sap domain-containing proteins. Saposins and SAPLIPs employ various mechanisms for membrane binding, solubilisation, and fusion. Whereas binding primarily occurs through direct hydrophobic contacts, in some cases, it is facilitated or preceded by electrostatic targeting driven by positively charged residues which position the protein at the membrane surface. This is followed by rotation or conformational changes to expose hydrophobic interfaces for binding. For literature references, please see the main text.

For granulysin (PDB accession code 119l), Na-SLP-1 (3s63), saposins C (2qyp) and D (3bpq) and Ac-SLP-1 (3s64), the N- and C-terminal helices ( $\alpha 1$ and $\alpha 5$ ) are coloured blue, and helices $\alpha 2$ - $\alpha 4$ are orange. For the oligomeric species of saposin B (1n69) and amoebapore A (1fo9), these colours indicate different monomers. The proposed membrane-inserting amoebapore A hexamer is also rendered as surface representation with a colour ramp where red indicates high hydrophobicity. Protein structures were rendered with PyMOL. ${ }^{[94]}$

\section{Figure 3}

Comparison of Group 1 and Group 2 ASP structures, exemplified by Na-ASP-2 (PDB accession code 4nui) and Ac-ASP-7 (PDB accession code 3s6v). The N-terminal PR (CAP) domain is coloured blue, the C-terminal Hinge-like domain is rendered in red. Cysteine residues and disulphide bonds are shown as stick models in yellow. Group 1 ASPs (left) possess an equatorial groove that features a prominent tandem-histidine motif capable of binding metals (shown here is coordination of a $\mathrm{Co}^{2+}$ ion by His 88 and His148 and four water molecules). This groove is absent from Group 2 ASPs (right) due to absence of a linker of $\sim 25$ amino acids length between the two $\alpha$ helices on the left hand side, which also results in absence of a disulphide bond. The Hinge-like domain of Ac-ASP-7 can bind a variety of metal ions; shown here is a $\mathrm{Mn}^{2+}$ ion. Protein structures were rendered with PyMOL. ${ }^{[94]}$ 


\section{Figures}

\section{Figure 1}

Na-SLP-1

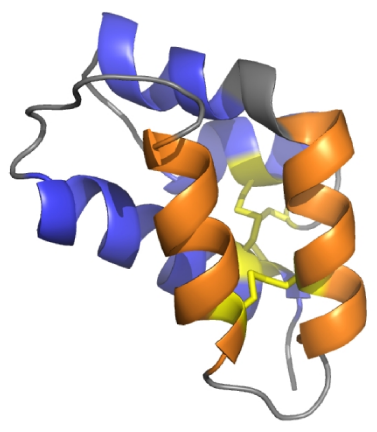

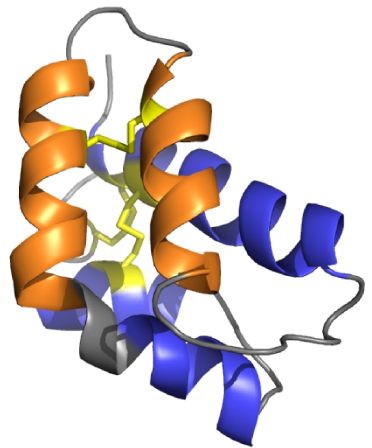

Ac-SLP-1

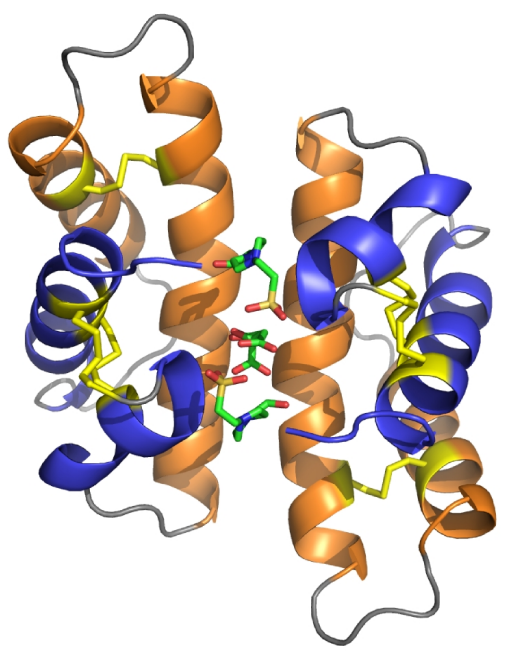

Crystal structures of Na-SLP-1 (PDB accession code 3s63) and Ac-SLP-1 (PDB accession code 3s64). The topology of the Sap domain fold is highlighted by the blue/orange colouring. Disulphide linkages are rendered as stick models and coloured yellow. Both structures reveal dimeric arrangements with interface areas of $571 \AA^{2}$ and $767 \AA^{2}$, respectively. As suggested by the rather small interface area observed in the $N a$-SLP-1 crystal structure, this protein exists exclusively as monomer in solution. Ac-SLP-1, in contrast, is dimeric in solution. The dimer interface of Ac-SLP1 features a long groove in which a citrate and two HEPES molecules were found in P2-symmetric positions. The citrate molecule occupies a special position and only one conformation is shown for clarity. Protein structures were rendered with PyMOL. ${ }^{[94]}$ 


\section{Figure 2}

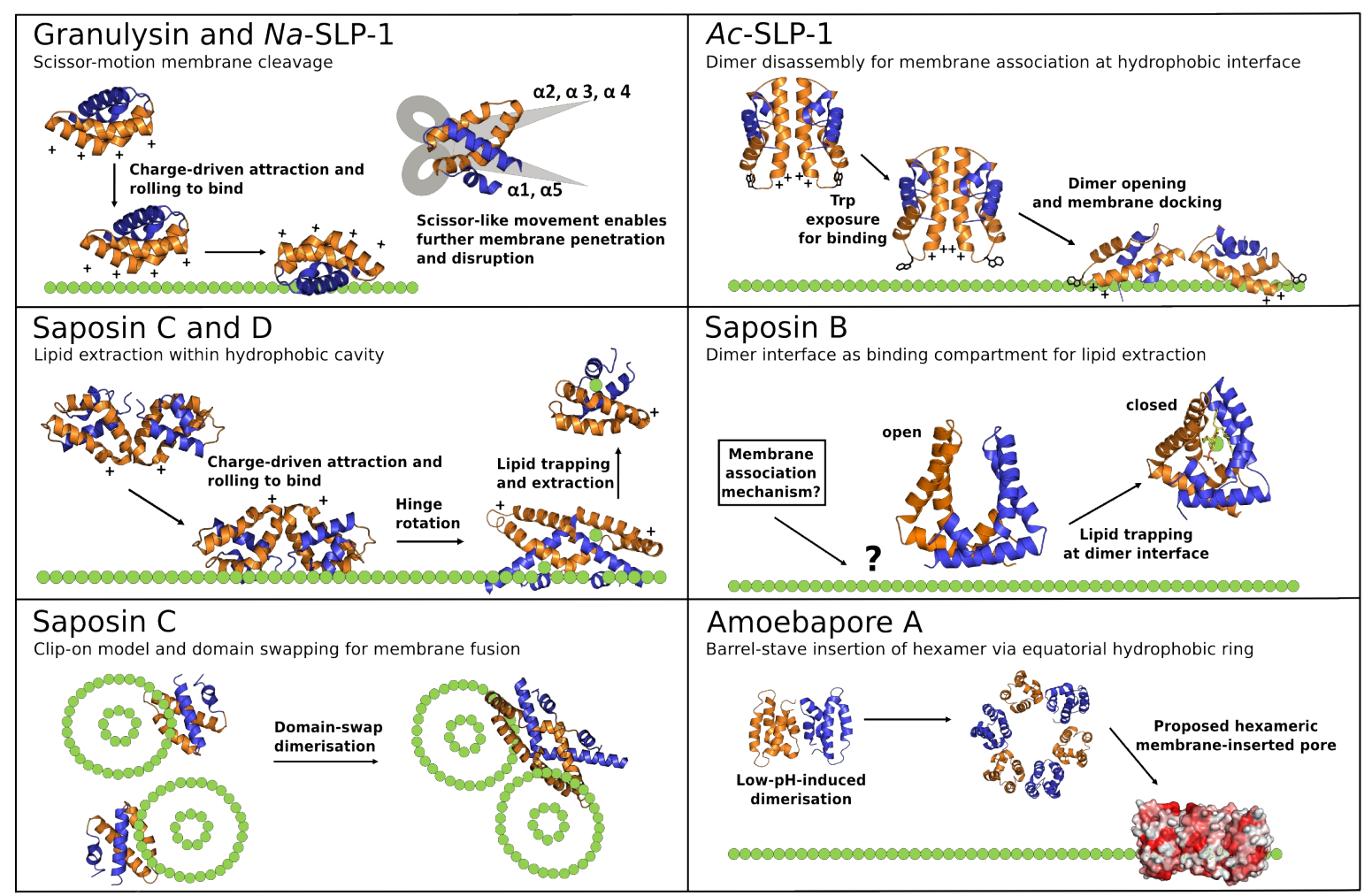

Versatile membrane interactions by Sap domain-containing proteins. Saposins and SAPLIPs employ various mechanisms for membrane binding, solubilisation, and fusion. Whereas binding primarily occurs through direct hydrophobic contacts, in some cases, it is facilitated or preceded by electrostatic targeting driven by positively charged residues which position the protein at the membrane surface. This is followed by rotation or conformational changes to expose hydrophobic interfaces for binding. For literature references, please see the main text.

For granulysin (PDB accession code 119l), Na-SLP-1 (3s63), saposins C (2qyp) and D (3bpq) and Ac-SLP-1 (3s64), the N- and C-terminal helices ( $\alpha 1$ and $\alpha 5$ ) are coloured blue, and helices $\alpha 2$ - $\alpha 4$ are orange. For the oligomeric species of saposin B (1n69) and amoebapore A (1fo9), these colours indicate different monomers. The proposed membrane-inserting amoebapore A hexamer is also rendered as surface representation with a colour ramp where red indicates high hydrophobicity. Protein structures were rendered with PyMOL. ${ }^{[94]}$ 


\section{Figure 3}

Group 1 ASPs: Na-ASP-2

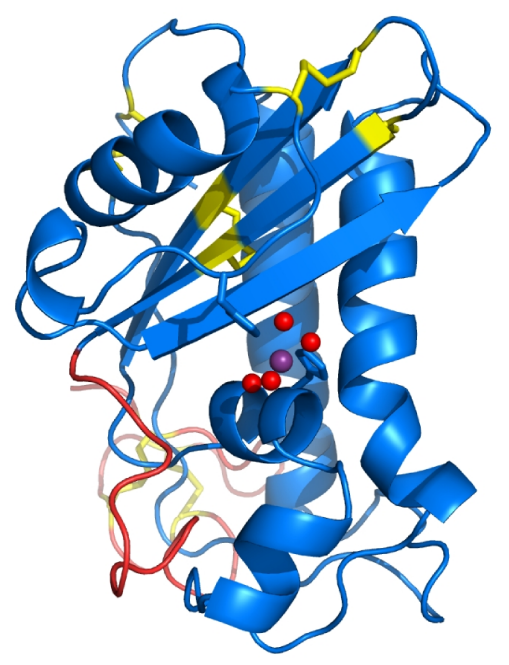

Group 2 ASPs: Ac-ASP-7

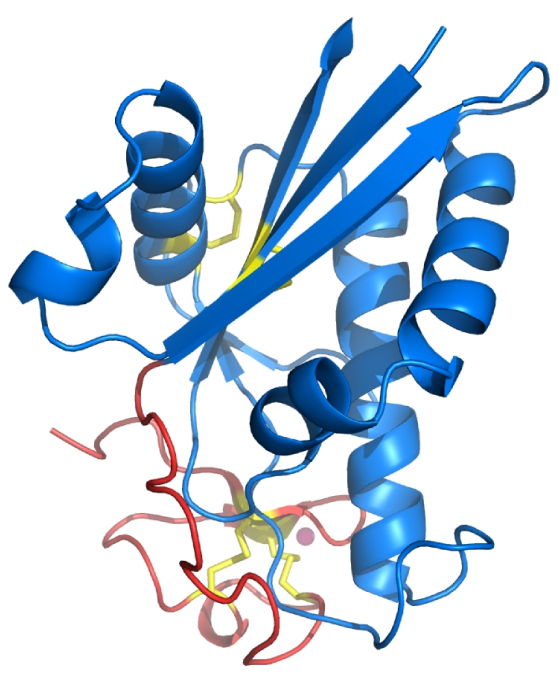

Comparison of Group 1 and Group 2 ASP structures, exemplified by Na-ASP-2 (PDB accession code 4nui) and Ac-ASP-7 (PDB accession code 3s6v). The N-terminal PR (CAP) domain is coloured blue, the C-terminal Hinge-like domain is rendered in red. Cysteine residues and disulphide bonds are shown as stick models in yellow. Group 1 ASPs (left) possess an equatorial groove that features a prominent tandem-histidine motif capable of binding metals (shown here is coordination of a $\mathrm{Co}^{2+}$ ion by His88 and His148 and four water molecules). This groove is absent from Group 2 ASPs (right) due to absence of a linker of 25 amino acids length between the two $\alpha$ helices on the left hand side, which also results in absence of a disulphide bond. The Hinge-like domain of Ac-ASP-7 can bind a variety of metal ions; shown here is a $\mathrm{Mn}^{2+}$ ion. Protein structures were rendered with PyMOL. ${ }^{[94]}$ 